\title{
Long term palliation of a metastatic primary gliosarcoma with stereotactic body radiotherapy
}

\author{
Janaki Manur Gururajachar, Sweta Polisetty
}

Department of Radiation Oncology, M. S. Ramaiah Medical College Bangalore, Karnataka, India

Received June 22, 2015; Revised November 27, 2015; Accepted December 03, 2015; Published Online December 23, 2015

Case Report

\begin{abstract}
Primary gliosarcoma (PGS) is rare and is known for extra cranial metastases. The overall outcome is dismal and hence the focus is on providing the best quality of life. This report describes a seventy-year-old lady who at presentation had metastatic primary gliosarcoma with intractable cough. Her lung lesion was treated with stereotactic body radiotherapy (SBRT) to a dose of 10 Gray per fraction for three consecutive days. She was free of cough till her death at eighteen months after treatment. This is probably the first reported case of excellent long-term quality of life in a patient of metastatic primary gliosarcoma.
\end{abstract}

Keywords: Gliosarcoma; Lung Metastases; SBRT

\section{Introduction}

Gliosarcoma (GS) constitutes $1.8-2.8 \%$ of all glioblastoma multiforme (GBM). ${ }^{1}$ It can be primary when it occurs "de novo" as opposed to secondary when initially the patient was treated as glioma and subsequently the recurrence reveals gliosarcoma. ${ }^{2}$ In addition to histologically resembling GBM, it also shows mesenchymal component which resembles fibroblast, cartilage, bone, smooth muscle, striated muscle or adipose tissue. ${ }^{3}$ Due to the sarcomatous component, they are prone for extracranial metastasis, most common sites being liver, lung and bone. ${ }^{4}$ The mesenchymal element is the reason for its poor prognosis compared to GBM. The treatment is same as that of GBM which includes maximum safe resection followed by adjuvant radiation and temozolamide. ${ }^{5}$ From a single institute in India, Kumar et al. ${ }^{6}$ conducted an audit of 24 patients and found that the overall survival was similar, 7.3 and 7.5 Months with GS and GBM respectively. Maximum time to disease progression is 11 months and longest survival described in the literature is 18 months. $^{5}$

Majority of the times, the emphasis is on the cancer directed therapy and relatively less importance is given to the worsening symptoms. Also the therapy itself has its own acute sequelae that may add to the already compromised quality of life. Hence, it is very important to employ treatments that are more effective and least toxic. Hypo fractionation with stereotactic body radiotherapy (SBRT) delivers high dose in short time with minimal toxicity. This is an attempt to present a case report of a metastatic primary gliosarcoma who had complete relief from cough until her death.

\section{Methods and Materials}

A 73-year-old lady presented with weakness of right hand of 20 days duration. She was diabetic since last ten years, hypertensive since one year and asthmatic for past five years and was on regular medication. She was investigated with magnetic resonance imaging (MRI) and underwent gross total excision of a space-occupying lesion arising from the left parietal region. The postoperative histopathology was grade IV Gliosarcoma.

Patient was taken up for adjuvant radiation 40 Gy/20 fraction with 3-dimensional conformal radiotherapy technique (3DCRT) along with temozolamide. Patient had persistent cough and hence a chest x-ray was done which revealed a lesion in the midzone of right lung. The cough was persistent and the patient was not able to sleep which severely compromised her quality of life. Patient was subsequently taken up for SBRT, boost to the tumor bed and palliative radiation to the lung lesion. The planning positron emission tomography (PET) scan picked up additional lesions in the left gluteal region and right upper thigh, both were asymptomatic and hence kept under observation.

Corresponding author: Janaki Manur Gururajachar; Department of Radiation Oncology, M. S. Ramaiah Medical College Bangalore, Karnataka, India.

Cite this article as: Manur Gururajachar J, Polisetty S. Long term palliation of a metastatic primary gliosarcoma with stereotactic body radiotherapy. Int J Cancer Ther Oncol 2016; 4(1):414. DOI: 10.14319/ijcto.41.4 


\subsection{Stereotactic body radiotherapy}

Three gold fiducials were placed in and around the lung lesion and radiotherapy planning was done seven days later. Patient was immobilized with orifit cast for the head and vacloc for the lung treatment. Simulation was done using computerized tomography (CT), MRI as well as PET scan. Images of CT /MR were fused for contouring brain lesion and CT/PET images were used to contour lung lesion. PET detected lung lesion was contoured as gross tumour volume. Spinal cord, lungs, heart, great vessels, esophagus and tracheobronchial tree were contoured as organs at risk. A plan was generated on multi plan treatment planning system (TPS) using ray tracing algorithm. The dose received by the tumour as well as normal structures (Figure 1) was evaluated qualitatively in axial, coronal and sagittal sections (Figure 2-4) and quantitatively using dose volume histograms (Figure 5). Patient was treated to a dose of 10 Gy per fraction prescribed at $82 \%$, for consecutive three days. A total of 247 beams were used at 87 different nodal stations as shown (Figure 6). Intrafraction verification was done tracking the fiducials and synchrony system was used to monitor respiration. Patient had severe cough during the first fraction, the treatment had to be interrupted many times and hence took a longer time. However, subsequent fractions were uneventful. Patient also received boost dose of radiation to the brain lesion to a dose of $12 \mathrm{~Gy}$ in two fractions.

\begin{tabular}{|c|c|c|c|c|c|c|c|}
\hline \multicolumn{2}{|c|}{ Nodes } & \multicolumn{2}{|c|}{87} & \multicolumn{3}{|c|}{ Total MU } & 47534.05 \\
\hline \multicolumn{2}{|c|}{ Beams } & \multicolumn{2}{|c|}{247} & \multicolumn{3}{|c|}{ Min MU } & 20.43 \\
\hline \multicolumn{2}{|c|}{ Max Dose (cGy) } & \multicolumn{2}{|c|}{3658.54} & \multicolumn{3}{|c|}{ Max MU } & 397.79 \\
\hline \multicolumn{8}{|c|}{ Dose Statistics Table } \\
\hline VOI & $\begin{array}{l}\text { Min } \\
\text { (cGy) }\end{array}$ & $\begin{array}{l}\text { Mean } \\
\text { (cGy) }\end{array}$ & $\begin{array}{c}\operatorname{Max} \\
\text { (cGy) }\end{array}$ & $\mathrm{Cl}$ & $\mathrm{nCl}$ & $\mathrm{HI}$ & Coverage \\
\hline GTV Lung mets & 2499.43 & 3228.26 & 3658.54 & 1.43 & 1.45 & 1.22 & $98.47 \%$ \\
\hline outer 10 & 442.42 & 1374.01 & 2419.88 & nis & $n / a$ & nfa & nita \\
\hline outer_15 & 185.62 & 956.26 & 1616.60 & nis & Hiba & mirat & \\
\hline No_beam & 14.74 & 49.10 & 347.91 & nia & $\mathrm{n} / \mathrm{a}$ & $n / a$ & n \\
\hline Rt Lung & 37.12 & 693.94 & 3573.79 & nis & ilia & hred & \\
\hline Lt Lung & 17.19 & 118.99 & 871.33 & n/a & $n / a$ & nía & n \\
\hline Spinal cord & 39.19 & 81.45 & 287.84 & ma & inge & hras & \\
\hline Esophagus & 63.25 & 244.76 & 502.15 & $n / 8$ & $n / a$ & $n / a$ & n \\
\hline Tracheobronchie & 118.69 & 622.22 & 1635.97 & 173 & thas & mina & \\
\hline Great Vessels & 35.28 & 203.66 & 1139.90 & nia & $\mathrm{n} / \mathrm{a}$ & $n / a$ & n \\
\hline Heart & 15.85 & 264.29 & 2438.41 & 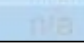 & 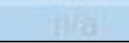 & 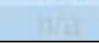 & \\
\hline
\end{tabular}

Figure 1: Table showing dose received by gross tumour and different organs at risk.

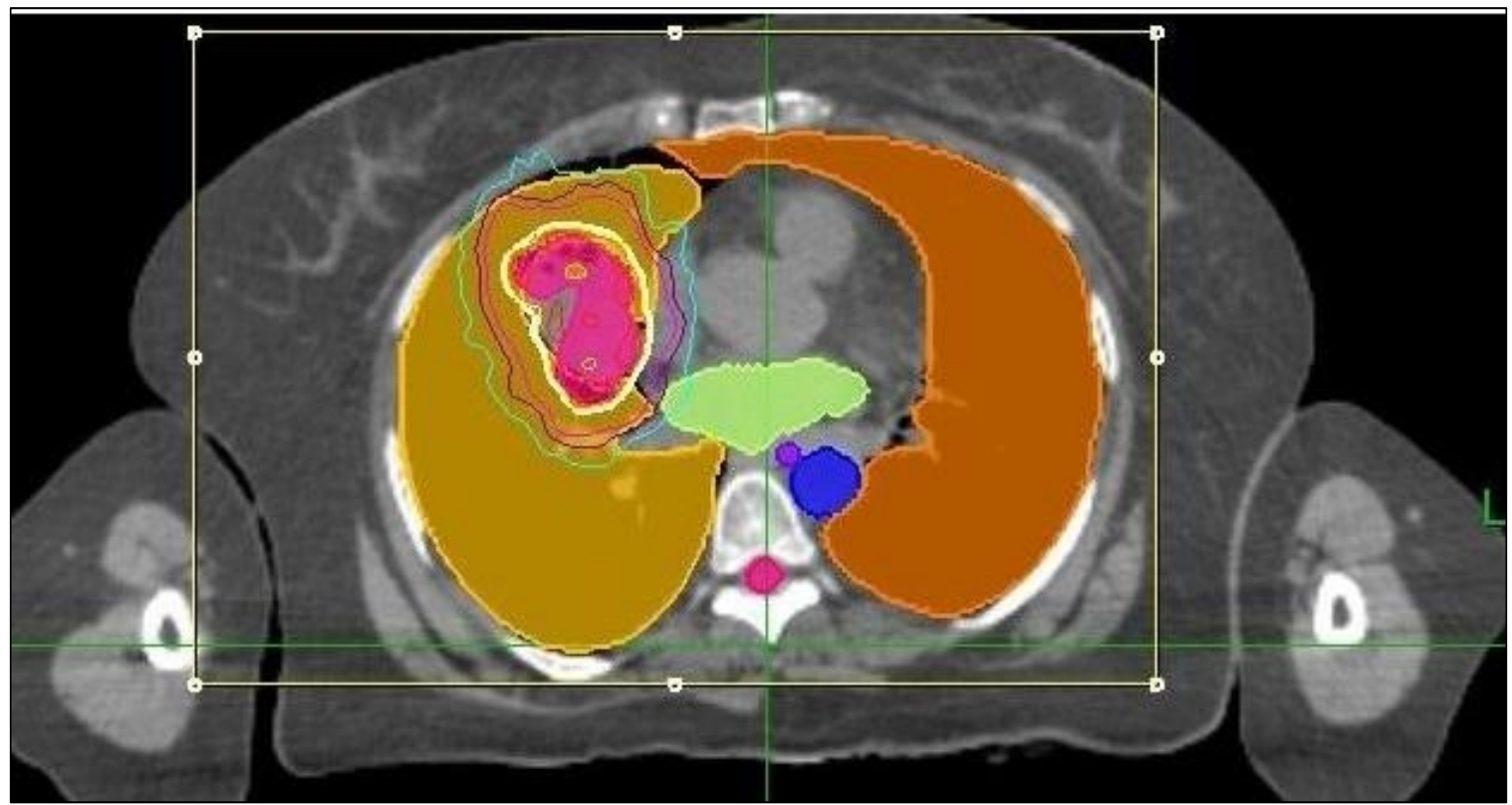

Figure 2: Axial section of the chest showing localized high dose to the tumour. 


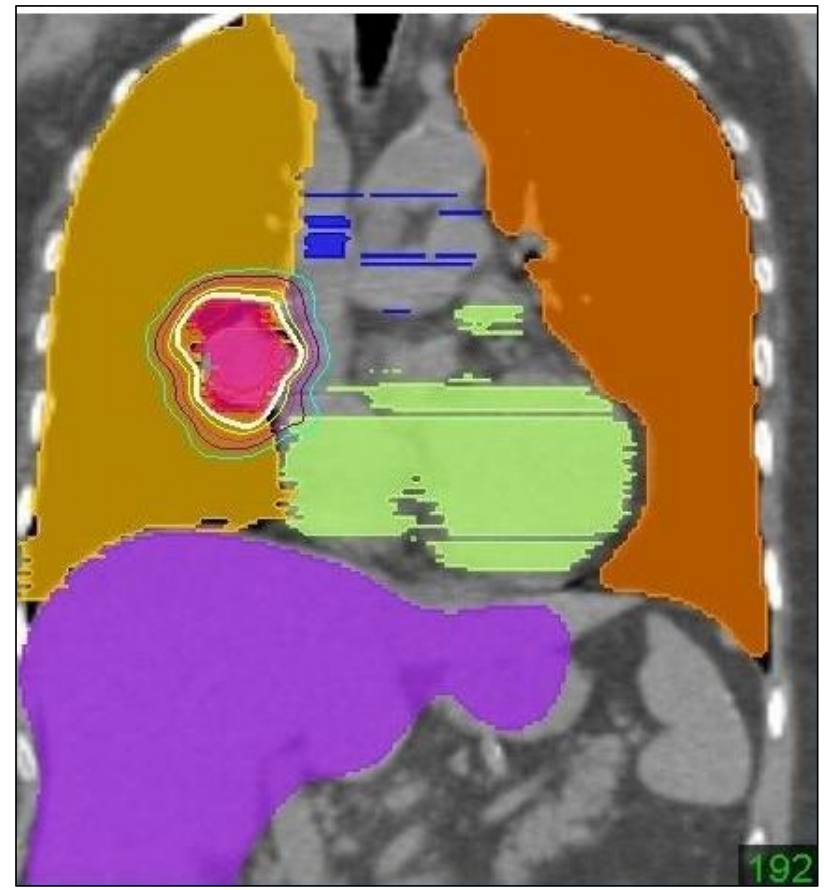

Figure 3: Coronal section of the chest showing dose distribution sparing the heart.

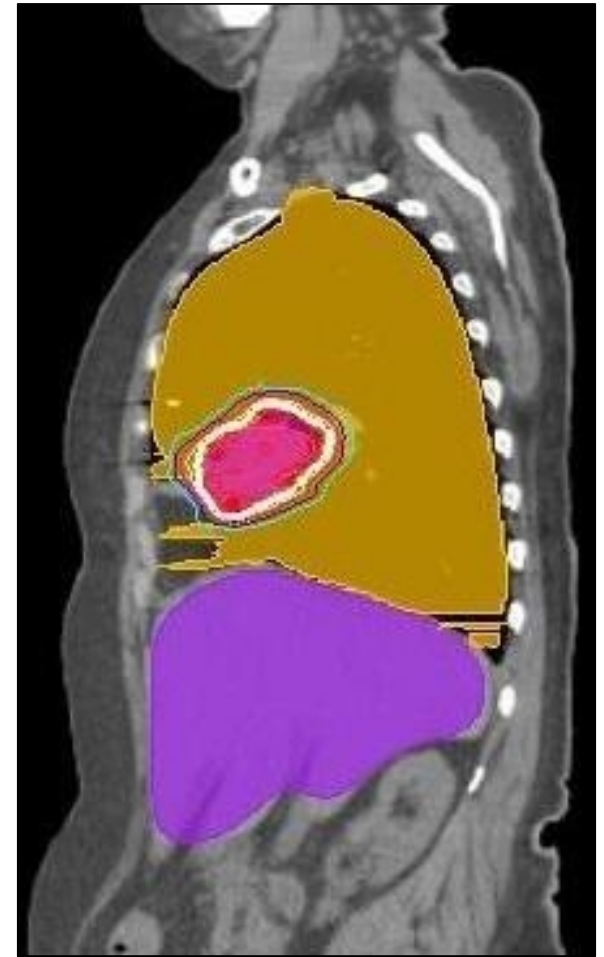

Figure 4: Sagittal section of the chest showing focused dose delivery sparing majority of the lung.

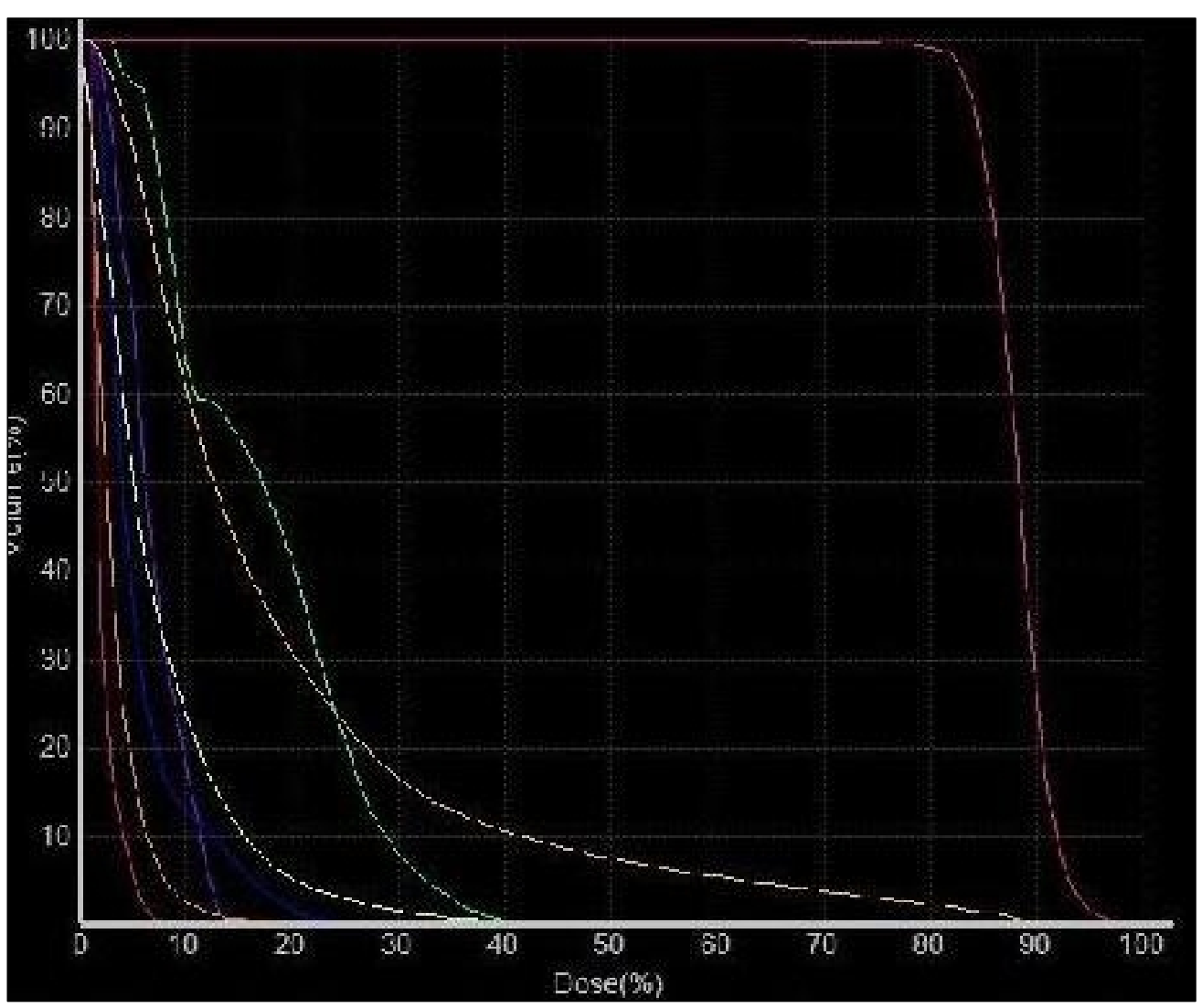

Figure 5: Dose volume histograms showing clear separation of the curves, normal structures on the left and tumour on the right. 


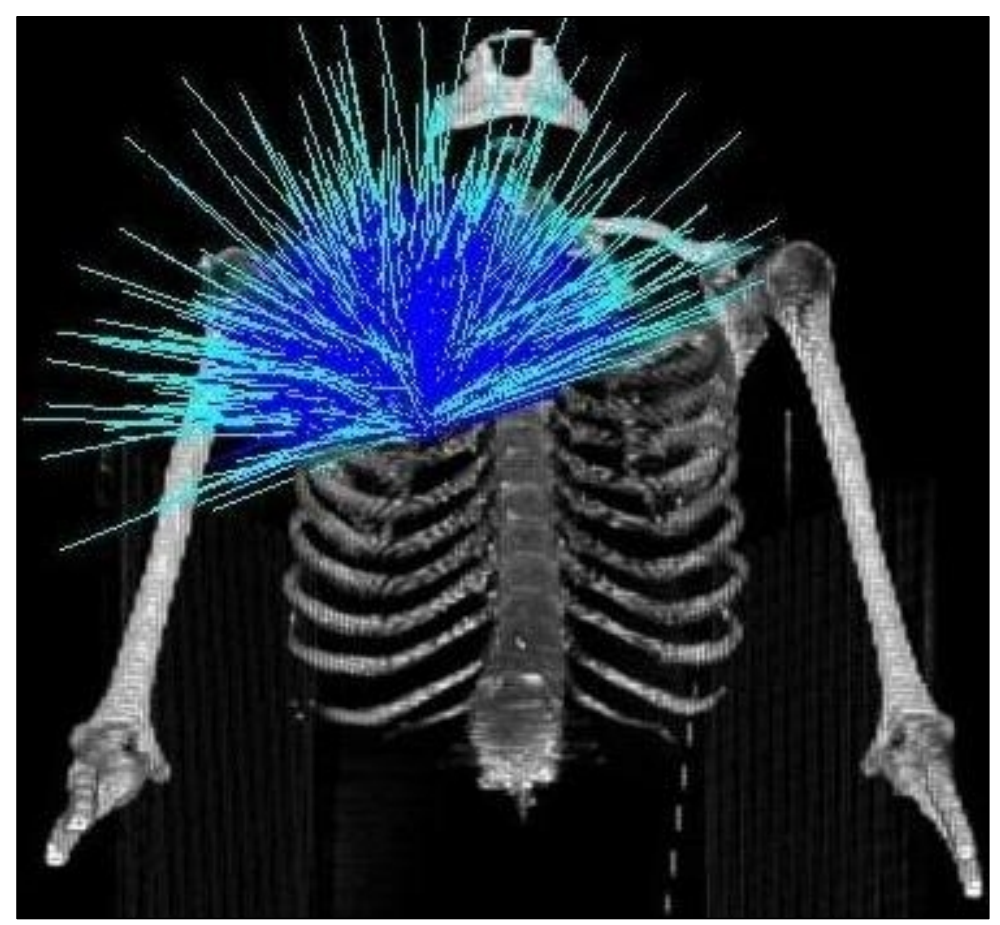

Figure 6: Three dimensional view of chest showing radiation beam entering from different angles.

\section{Results and Discussion}

She was on regular follow up and had complete relief from cough. PET scan done six months later showed reduction in lung lesion size, however the soft tissue lesions had increased in size but patient still remained asymptomatic. She was alive for 18 months after diagnosis when she died of myocardial infarction and was completely free of cough until her death.

Quality of life is the most important aspect of a metastatic disease. The emphasis to achieve this is on employing treatment modalities that are highly effective and at the same time less toxic for the patient. SBRT is one such radiation technique wherein multiple beams from different angles are used to focus on a particular point. Thus, it deposits maximum dose around that point and the surrounding normal structures get minimal dose, reducing the rate of complications. This is classically used in small tumours less than three cms, relatively radio resistant tumours, which require higher doses, and those tumours that are situated near critical structures. Our patient typically had all these factors and hence was treated with SBRT.

Cyber knife is a robotically controlled miniature linear accelerator with real time image guidance. Real time image guidance software and the robot in the cyber knife continuously tracks and adjusts for movement during the entire treatment. Multiple sets of orthogonal x-rays are taken, fiducials are tracked, and observed variations are corrected before the radiation is actually delivered. Different phases of breathing cycle are captured onto the computer with the help of fibre optic cables and light emitting diode's (LED) on the jacket that patient wears during treatment. The synchrony software identifies the tumour in relation to different phases of breathing cycle. Hence, it is possible to treat the tumour during a particular phase of breathing and there is no need of respiratory gating.

The long-term palliation of cough was due to the high dose delivered to the lesion and the reduction in size was documented with a subsequent PET scan. In a retrospective study, Christina et al have analyzed the role of SBRT for inoperable metastasis from primary sarcoma and have concluded that it is safe, convenient and effective and observed a median survival of 14.2 Months. $^{7}$

To our knowledge, this report is the first of its kind, which demonstrates long-term palliation of cough and is gratifying not only to the patient but also to the palliative caregivers.

\section{Conclusion}

Primary gliosarcoma is an aggressive tumour and is associated with a poor outcome. Cough as a symptom of metastatic cancer is very difficult to treat and reduces the quality of life of a terminally ill patient. Selected patients do have long-term palliation from SBRT which has the advantage of higher dose delivery over shorter duration of treatment and also is not associated with any complications. 


\section{Conflict of interest}

The authors declare that they have no conflicts of interest. The authors alone are responsible for the content and writing of the paper.

\section{References}

1. Kozak KR, Mahadevan A, Moody JS. Adult gliosarcoma: Epidemiology, naturalhistory, and factors associated with outcome. Neuro Oncol. 2009;11:183-91.

2. Andaloussi-Saghir K, Oukabli M, El Marjany M, et al. Secondary gliosarcoma after the treatment of primary glioblastoma multiforme. N Am J Med Sci. 2011;3:527-30.

3. Miller CR, Perry A. Glioblastoma. Arch Pathol Lab Med. 2007;131:397-406.

4. Beaumont TL, Kupsky WJ, Barger GR, Sloan AE. Gliosarcoma with multiple extracranial metastases: Case report and review of the literature. J Neurooncol. 2007;83:39-46.
5. Han SJ, Yang I, Tihan T, et al. Primary gliosarcoma: Key clinical and pathologic distinctions from glioblastoma with implications as a unique oncologic entity. J Neurooncol. 2010;96:313-20.

6. Kumar P, Singh S, Kumar P, et al. Gliosarcoma: An audit from a single institution in India of 24 post-irradiated cases over 15 years. J Cancer Res Ther. 2008;4:164-8.

7. Stragliotto CL, Karlsson K, Lax I, et al. A retrospective study of SBRT of metastases in patients with primary sarcoma. Med Oncol. 2012;29:3431-9. 